\begin{tabular}{|c|c|c|}
\hline Beitr. Ent. & Keltern & ISSN 0005-805X \\
\hline $53(2003) 1$ & S. $231-238$ & 31.07 .2003 \\
\hline
\end{tabular}

\title{
Zur Identität der aus Madagaskar beschriebenen Autoserica stupida BRENSKE, 1900
}

\section{(Coleoptera: Scarabaeidae: Sericini)}

Mit 8 Figuren

\section{DIRK AHRENS}

\section{Zusammenfassung}

Eine Revision des Typenmaterials der von Madagaskar beschriebenen Autoserica-Arten bestätigte die artliche Übereinstimmung von Autoserica stupida BRENSKE, 1900 mit der orientalischen Maladera straba (BRENSKE, 1898). Die folgenden neuen Synonyme und Kombinationen wurden festgestellt: Maladera straba (BRENSKE, 1898) comb. n. (= Neoserica apogonoides BRENSKE, 1898, syn. n., = Autoserica stupida BRENSKE, 1900, syn. n., = Neoserica sumatrensis BRENSKE, 1898, syn. n., = Neoserica pavieana BRENSKE, 1899, syn. n.), Maladera detersa (ERICHSON, 1834) comb. n. Die systematische Position und aktuelle Verbreitung der Arten wird diskutiert. Für $M$. detersa (ERICHSON) wird ein Lectotypus festgelegt.

\section{Summary}

A revision of the type material of species described as Autoserica BRENSKE, 1897 from the Malagasy region confirmed the conspecificity of Autoserica stupida BRENSKE, 1900 with the Oriental species Maladera straba (BRENSKE, 1898) comb. $n$. The following four new synonyms and two new combinations are established: Maladera straba (BRENSKE, 1898) comb. n. (= Neoserica apogonoides BRENSKE, 1898, syn. n., = Autoserica stupida BRENSKE, 1900, syn. n., = Neoserica sumatrensis BRENSKE, 1898, syn. n., = Neaserica pavieana BRENSKE, 1899, syn. n.), Maladera detersa (ERICHSON, 1834) comb. n. The systematic position and distribution of the species are discussed. A lectotype is designated for M. detersa (ERICHSON).

\section{Keywords}

Coleoptera, Scarabaeidae, Sericini, Madagaskar, Oriental region, new synonymy, new combinations, distribution

\section{Einleitung}

Im Rahmen einer Revision der madegassischen Vertreter der Sericini, die unter dem Gattungsnamen Autoserica BRENSKE, 1897 beschrieben wurden, ist eine weitere orientalische Art festgestellt worden, die ursprünglich von Madagaskar beschrieben wurde (BRENSKE 1900). Nachdem bereits für Maladera affinis (BLANCHARD, 1850) eine offensichtliche Ver- 
schleppung von Indien nach Madagaskar und Reunion diskutiert wurde (AHRENS im Druck b), ist in einem anderen Fall für eine urspünglich aus Madagaskar beschriebene Art, Oxyserica pygidialis BRENSKE, 1900, ein Vorkommen für Madagaskar ausgeschlossen und eine Fundortverwechslung angenommen worden (AHRENS im Druck a).

Da nur sehr wenige Taxonomen regionenübergreifend arbeiten, erschien es dem Autor als sinnvoll, in dieser separaten taxonomischen Abhandlung den Status, die Synonymie und die reelle Verbreitung der aus Madagaskar beschiebenen Autoserica stupida BRENSKE, 1900 aufzuklären.

\section{Material und Methoden}

Zur Identifizierung der Tiere wat es notwendig, von allen $o^{*} \sigma^{x}$ die Genitalien zu untersuchen. Dazu wurde bei den sehr alten Exemplaren das Abdomen entfernt und der Aedoeagus über die basale Seite aus dem Abdomen herauspräpariert. Sie wurden anschließend mit dem Käfer auf den gleichen Karton geklebt. Die Zeichnungen sowie Längenmessungen wurden mit einem Carl-Zeiss Jena Stereomikroskop vom Typ SM20 mittels Okularmikrometer angefertigt.

\section{Abkürzungen}

$\begin{array}{ll}\text { BPBM } & \text { Bernice P. Bishop Museum, Honolulu } \\ \text { CA } & \text { Coll. Dirk Ahrens, Eberswalde } \\ \text { CF } & \text { coll. G. Frey (im NHMB) } \\ \text { DEI } & \text { Deutsches Entomologisches Institut, Eberswalde } \\ \text { HNHM } & \text { Hungarian Natural History Museum, Budapest } \\ \text { MHNG } & \text { Muséum d'Histoire Naturelle, Genf } \\ \text { MNHN } & \text { Museum National d'Histoire naturelle, Paris } \\ \text { NHMB } & \text { Naturhistorisches Museum, Basel } \\ \text { SMTD } & \text { Staatliches Museum für Tierkunde, Dresden } \\ \text { ZMHB } & \text { Zoologisches Museum der Humboldt-Universität, Berlin }\end{array}$

Maladera straba (BRENSKE, 1898) comb. $\mathrm{n}$.

(Fig. 1-5)

Autoserica straba BRENSKE, 1898: 277; ARROW 1916: 431.

Neoserica apogonoides BRENSKE, 1898: 380; Autoserica apogonoides: MOSER 1915: 356, syn. n.

Neoserica sumatrensis BRENSKE, 1898: 395; Autoserica sumatrensis: MOSER 1915: 356, syn. n.

Neaserica pavieana BRENSKE, 1899: 416; BRENSKE 1902: 65, syn. n.

Autoserica stupida BRENSKE, 1900: 52, syn. n.

Typen-Material: Syntypen (straba): 3 o $^{\star}, 1$ \% „Ceylon/ straba type Brsk./ Coll. Brenske“" (ZMHB), 1 o" „Ceylon/ Coll. C. Felsche Kauf 20, 1918/ straba type Brsk." (SMTD). Syntypen (apogonoides): 10 „Singapore/Collection Mniszeck“ (MNHN), 2 o o ",Singapore 6.1865 G. Doria“" (MSNG, DEI), 1 † „Singapore Doria.65/ apogonoides'Type Brsk./ coll. Brenske" (ZMHB), 2 ơ ơ, 1 o "Singapore Doria.65/ apogonoides Brsk./ coll. Brenske" 
(ZMHB), $1 \sigma^{7}$ „Singapore Doria.65// apogonoides Type Brsk./ E. Brenske 1896/ Musuem Paris ex Coll. R. Oberthur/ Typus“ (MNHN), $10^{\star}$ „Singapore Doria.65/ Gestro donatus/ Museum Patis Singapoure Brenske 1898/ Cotype/ Serica apogonoides cotype Brsk." (MNHN), 2 우 ㅇ ,Singapore Doria.65/ 9 / apogonoides Brsk./ E. Brenske 1896/ Musuem Paris ex Coll. R. Oberthur" (MNHN), $70^{\circ}$ o, 14 ㅇ $q$ „Singapore Doria.65." (MSNG, ZMHB), 1 \% "Singapore Doria.65./ Museo Civico di Genova/ Serica apogonoides Brsk." (MSNG), $10^{\star}$ "Singapore/Collectio Mniszeck/Ex Museo van Lansberge/ apogonoides type Brsk./ E. Brenske 1896/ Museum Paris Ex Coll. R. Oberthur" (MNHN), 1 \% ,Malais/Ex. Museo Mniszeck/apogonoides Brsk./ E. Brenske $1896 /$ Musuem Paris ex. Coll. R. Oberthur" (MNHN), $10^{\star}$ „Singapore Deyt. v./ apogonoides Brsk./ coll. Brenske/" (ZMHB), 1 q "Malay Pen/ coll. Brenske/ apogonoides Brsk." (ZMHB), 2 ㅇ ㅇ "Malakka coll. Brenske/ apogonoides Brsk." (ZMHB), 1 i ,Malacca/ Serica apogonoides Brenske" (DEI), 1 o "Serica apogonoides Malacca Deyr./ apogonoides type Brsk./ coll. Kraatz" (DEI), $1 \sigma^{\circ}$ "Singapore/ coll. Brenske/ apogonoides type Brsk." (ZMHB), $2 \sigma^{*} \sigma^{*}, 63395 /$ Singapore Hilgendorf/ apogonoides Brsk." (ZMHB), $5 \sigma^{*} \sigma^{*}, 1$ o „57256/ Singapore Engel“ (ZMHB), 1 o ,3159/ apogonoides Type Brsk." (ZMHB). Syntypus (stupida): $10^{\star}$,Madagascar/ Serica stupida Typ. Brsk./ Coll. Brenske/ stupida Brsk." (ZMHB). Syntypen (sumatrensis): $3 \sigma^{\star} \sigma^{\star}, 2$ \& $q$,N.O. Sumatra Tebing-tinggi Dr. Schultheiss/ coll. Kraatz" (DEI), 10 , 3 q $q$ „N.O. Sumatra Tebing-tinggi Dr. Schultheiss" (ZMHB) Syntypen (paviena): 1 \% ,Museum Paris Cambodge Pnom-Penh A. Pavie $1886 /$ Neaserica paviena type 9 Brsk./ Type“ (MNHN), $10^{*}$ „Museum Paris Cambodge Battambang A Pnom Penh A. Pavie 1886/Neoserica paviena Type $q$ Brsk./Type" (MNHN).

Weiteres Material: 1 ex. „Malacca Singapore“ (CF), 2 ex. „Rajang Selangore 97-203“ (CF), 1 ex. (đ) „Malaya Doherty/ coll. Chev./ filitarsis Chev. Malacca/ coll. Brenske“ (ZMHB), 2 ex. „Singapore/ Collectio Dr. Ohaus" (SMTD), 1 ex. ,Malaya: Pahang Bukit Ibam, $90 \mathrm{~km}$ WNW of Kuala Rompin ca. 50 m, 4.9.X.1961/ K.J. Kuncheria Collector Bishop" (BPBM), 1 ex. ( $\left.{ }^{\star}\right)$, W'est Malaysia, Ostküste: Rantau Abang, Strandnähe, am Licht 21.7.1981, Hendrich leg.“ (CA), 1 ex. „Malaisie Johor Sediti Kechil Kota Tinggi 10-13.VI.69 R. Pilet" (MHNG), 10 ex. „Malaisic Selangor Sungei Buloh Kuala Lumpur 20-IX-72 T. Jaccoud" (MHNG),

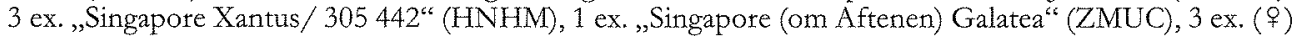
"Naer Singora Syd-Siam Oktb. 1940 A.M. Hemmingsen" (ZMUC), 1 ex. (ð) „Museum Paris Lakhon Harmand 1878/ 163 78/ Mt. Lakhon Harmand“ (MNHN), 2 ex. „Museum Paris Presou de Malacca Errington de la Croix et P. Chape 1899" (MNHN), 10 ex. „Serdang, Selangor F.M.S. IX.I.30/C.E. Pemberton Collector/ F.C. Hadden Collection" (BPBM), 1 ex. ( $\%)$ "Sumatra - Indonesien Tebing/ Tingyi (Deli) leg.

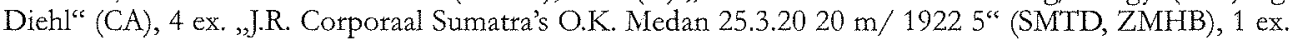
"Corporaal Sumatra Medan 4-1920" (ZMHB), 7 ex. "Sumatra OK Asahan 50-60 m Goenoeng Malajoe Gambirpflanzung/ Schneider 1934-36/ 6526e/ Sammlung Fritz Schneider Wadenswill 1997" (NHMB), 1 ex. $\left(0^{*}\right)$ "Medan-Deli. 300'ffeet] 2de Sem. 89 I.Z. Kannegieter" (CA), 1 ex. „Sumatra/ 28.T 84." (DEI), 1 ex. "Sumatra Deyrolle/ $40805^{\prime \prime}$ (ZMHB), 6 ex. „Bedagei int. Sumatra's O.K. 600' 2 de Sem.89 I.Z. Kannegieter" (ZMHB), 4 ex. „Edi Klein“ (ZMUC), 4 ex. „Holl. Indien Sumatra Klein“ (ZMUC).

Fragliche Fundortangabe: 11 ex. „Conchinchina/ coll. Brenske" (ZMHB), $10^{\star}, 2$ 우 "Conchinchina/ coll. Brenske/ detersa" (ZMHB), 1 ex. ( $\left.\sigma^{*}\right)$ "Conchinchina/ coll. Brenske/ detersa Er. type vergl." (ZMHB).

Länge: 6,6-8,1 mm, Elytrenlänge: 4,8-5,0 mm, Breite: 3,9-4,1 mm. Körper oval; einfarbig, rötlich braun bis gelbbraun; Oberseite stark glänzend, bis auf wenige einzelne Haare auf dem Kopf, den lateralen Elytrenintervallen und die Seitenrandbehaarung von Pronotum und Elytren kahl, Tarsen, Tibien und Labroclypeus ebenfalls glänzend. 
Labroclypeus kurz und mäßig breit, quer trapezoid, an der Basis am breitesten, Seitenränder kaum gebogen nach vorn verengt, durch einen nur sehr undeutlichen Winkel vom Ocularcanthus abgesetzt, am Übergang des Clypeus zum Labrum Seitenrand nicht eingeschnitten, Ränder schwach aufgebogen, Vorderwinkel breit abgerundet, Vorderrand median leicht konkav ausgerandet; Oberfläche in der Mitte leicht konvex, fein und dicht bis sehr dicht punktiert, im vorderen Drittel mit auf jeder Seite mit einem einzelnen, langen feinen Haar. Frontoclypealnaht sehr fein eingeritzt und kräftig gebogen. Augenspiegel wenig breit und kurz, glatt, etwa 1,5mal so breit wie lang, Ocularcanthus kurz und breit, glatt, mit einer langen Terminalborste. Stirn fein und sehr locker punktiert, neben dem Augenspiegel mit einzelnen langen, abstehenden Haaren. Augen klein, Verhältnis: Durchmesser/Abstand: 0,58. Antenna 10-gliedrig, Clavus 3-gliedrig, bei beiden Geschlechtern etwas kürzer als die übrigen Glieder zusammen und gerade. Mentum konvex erhaben, vorn etwas abgeplattet; Labrum nach vorn stark verschmälert.

Pronotum kurz hinter der Mitte am breitesten, Seitenränder gleichmäßig und kräftig gebogen, nach vorn stärker verengt, Hinterwinkel stumpf, in der Spitze schwach abgerundet, Vorderecken spitz und deutlich vorgezogen, Seitenrand unmittelbar neben den Vorderwinkeln nur sehr schwach konvex, Vorderrand kräftig gerandet, nicht konvex vorgezogen, wie der Seitenrand locker und kräftig behaart; Oberfläche dicht und fein punktiert, ohne mikroskopische Behaarung in den Punkten. Scutellum breit dreieckig, fein und mäßig dicht punktiert, längs der Mitte ohne glatte Linie.

Elytren im hinteren Drittel am breitesten, Außenwinkel an der Spitze breit abgerundet, Streifen mäßig, Nahtstreifen etwas kräftiger eingedrückt und fein, dicht punktiert, Intervalle eben, fein und dicht punktiert, äußere Intervalle und vereinzelt auch die mittleren mit wenigen einzeln stehenden, langen, abstehenden Haaren, an der Schulterbeule in Nähe der Epipleuralkante mit einem einzelnen, langen Haar, in der Punktur sonst nur mikroskopisch behaart; Spitzenrand häutig, mit schmalem Saum aus feinen Mikrohärchen; Epipleuralkante kräftig, endet an der äußeren Spitzenrundung der Elytren, Epipleuren bis zur äußeren Spitzenrundung kurz und mäßig dicht behaatt.

Unterseite glänzend und kräftig, mäßig dicht punktiert, nur spärlich behaart, mit wenigen kräftigen Haaren auf Mesosternum und Metasternalplatte, Metacoxalplatten außen mit einigen kräftigen Haaren, Abdominalsternite mit je einer Querreihe haartragender Borstenpunkte zwischen der übrigen feinen, zerstreuten Punktur, vorletztes Sternit in der Mitte mit schmalem chitinösen Saum; Mesosternum zwischen den Mesocoxae etwas breiter als der Metafemur. Verhältnis: Metepisternen-/Metacoxalänge: 1/1,88, Außenwinkel der Metacoxalplatten nicht abgerundet, Hinterrand gerade. Pygidium apikal bei beiden Geschlechtern schwach gewölbt, fein und mäßig dicht punktiert, am Apikalrand einzeln, lang behaart, sonst mikroskopisch behaart.

Mesofemur und Metafemur glänzend und mit zwei Längshaarpunktreihen, dazwischen nur sehr spärlich fein punktiert, Metafemur vorn scharf gekantet und breit, neben dem Vorderrand ohne krenulierte Linie, Punkte der vorderen Längspunktreihe einfach, deren Ränder nicht krenuliert, Punkte der hinteren Längshaarpunktreihe dicht und kräftig, Hinterrand fast gerade, kahl, apikal weder ventral noch dorsal krenuliert, dorsoapikal zur Aufnahme der Tibia breit deckenartig produziert. Mesotibia an der Ventralkante nur mit zwei feinen Borsten. Metatibien kurz und breit, in der Mitte am breitesten, basal verschmälert, Verhältnis: Breite/Länge: 1/2,4; dorsal scharf gekantet, mit zwei kräftigen 
Außendorngruppen, die basale kurz vor der Mitte, die apikale bei etwa 3/4 der Metatibienlänge, basal davor eine weitere kräftige Borste aber ohne krenulierte Leiste; außen schwach längsgewölbt, lateral sehr fein und spärlich punktiert und sonst kahl; Ventralkante mit fünf gehöckert stehenden, langen Borsten, die den gleichen Abstand zueinander haben; Innenseite völlig glatt und kahl, ventroapikad an der Tarsaleinlenkung seicht konkav ausgeschnitten. Tarsen dorsal unpunktiert, ohne Seitenleisten, Metatarsen ventral mit krenulierter Leiste und ventral kahl, unmittelbar daneben eine weitere kräftige Längskante, Metatarsomer 1 etwas kürzer als die zwei folgenden Segmente zusammen und nur wenig länger als der obere Enddorn der Metatibien; Protibia sehr kurz, 2zähnig. Alle Klauen normal, symmetrisch.

Aedoeagus: Fig. 1-5.

Bemerkung: Die Syntypen der Maladera straba sind im Bau der Parameren identisch mit dem der Autoserica apogonoides, Neoserica sumatrensis und Autoserica stupida (siehe Fig. 15). Die nominellen Taxa sind daher als Synonyme aufzufassen. Da dem Autor nach intensiver Suche im diversen Sammlungen der großen naturhistorischen Museen keinerlei Material dieser Art aus Madagaskar vorlag, ist davon auszugehen, dass es sich bei der Fundortangabe der Autoserica stupida „Madagascar" um eine Fundortverwechslung handelt. Es wäre nicht die erste Fundortverwechslung im Material von Chevrolat in der Sammlung des ZMHB (siehe Oxyserica pygidialis BRENSKE, 1902 - AHRENS in Druck a). Gegenwärtig ist $M$. straba lediglich von der Malayischen Halbinsel und Sumatra sicher belegt. Von Sri Lanka liegen nur die alten Funde des Typenmaterials vor.

Maladera straba (BRENSKE) ist eng mit Maladera detersa (ERICHSON, 1834) verwandt, mit der sie zwei wichtige Synapomorphien teilt: rechte Paramere apikal kreisförmig ausgerandet und Körperoberfläche glänzend. Da letztere nur diesen beiden Arten gemeinsam ist, dürfte zwischen beiden Taxa ein Schwesterngruppenverhältnis anzunehmen sein. In der äußeren Morphologie sind beide Arten nur sehr schwer zu unterscheiden.

\section{Maladera detersa (ERICHSON, 1834) comb. n.}

(Fig. 6-8)

Serica detersa ERICHSON, 1834: 239.

Typen-Material: Lectotypus (hier festgelegt): ${ }^{*}$ „24901/detersa Er.* China Meyen.“ (ZMHB).

Weiteres Material: 44 ex. "Annam Phuc-Son Nov. Dez. H. Fruhstorfer" (ZMHB, DEI, CF, HNHM,

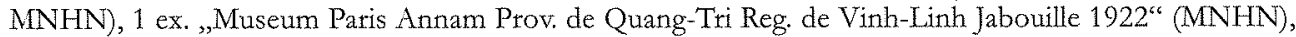
1 ex. „Süd-Annam Pha-Rang H. Fruhstorfer" (MNHN), 4 ex. "Tonkin Son Tai" (ZMHB), 1 ex. „Annam Phuc Son" (ZMHB), 2 ex. (o) "Dalat S. Vietnam" (CF), 20 ex. "Vietnam Da Nang 6-9.VII.1965/ malaise trap/A.T. Holway, Coll. Bishop Museum“ (BPBM), 5 ex. „VII-2. 1968 Kim Lien Republic of Vietnam Bruce W. Miller" (BPBM), 1 ex. „V-7. 1968 Kim Lien Republic of Vietnam Bruce W. Miller" (BPBM), 1 ex. „VI-21.1968 E. Danang Republic of Vietnam Bruce W. Miller“ (BPBM), 1 ex. „VIII-2.1968 E. Danang Republic of Vietnam Bruce W. Miller" (BPBM), 2 ex. „Coll. R. I. Sc. N. B. N. Vietnam: Tonkin Ngan Son Le Moult vendit“ (ISNB), 4 ex. (\#), Annam Laos" (HNHM).

Länge: 6,7-8,2 mm, Elytrenlänge: 4,8-5,0 mm, Breite: 3,9-4,2 mm. Körper oval; einfarbig, rötlich braun bis gelbbraun; Oberseite stark glänzend, bis auf wenige einzelne 
Haare auf dem Kopf, den lateralen Elytrenintervallen und die Seitenrandbehaarung von Pronotum und Elytren kahl, Tarsen, Tibien und Labroclypeus ebenfalls glänzend. Labroclypeus kurz und mäßig breit, quer trapezoid, an der Basis am breitesten, Seitenränder kaum gebogen nach vorn verengt, durch einen nur sehr undeutlichen Winkel vom Ocularcanthus abgesetzt, am Übergang des Clypeus zum Labrum Seitenrand nicht eingeschnitten, Ränder schwach aufgebogen, Vorderwinkel breit abgerundet, Vorderrand median leicht konkav ausgerandet; Oberfläche in der Mitte leicht konvex, fein und dicht bis sehr dicht punktiert, im vorderen Drittel mit auf jeder Seite mit einem einzelnen, langen feinen Haar. Frontoclypealnaht sehr fein eingeritzt und kräftig gebogen. Augenspiegel wenig breit und kurz, glatt, etwa 1,5mal so breit wie lang, Ocularcanthus kurz und breit, glatt, mit einer langen Terminalborste. Stirn fein und sehr locker punktiert, neben dem Augenspiegel mit einzelnen langen, abstehenden Haaren. Augen klein, Verhältnis: Durchmesser/Abstand: 0,62. Antenna 10-gliedrig, Clavus 3-gliedrig, beim fast so lang wie die übrigen Glieder zusammen und gerade, beim $\mathrm{E}$ etwas kürzer als die übrigen Glieder zusammen. Mentum konvex erhaben, vorn etwas abgeplattet; Labrum nach vorn stark verschmälert.

Pronotum kurz hinter der Mitte am breitesten, Seitenränder gleichmäßig und kräftig gebogen, nach vorn stärker verengt, Hinterwinkel stumpf, in der Spitze schwach abgerundet, Vorderecken spitz und deutlich vorgezogen, Seitenrand unmittelbar neben den Vorderwinkeln nur sehr schwach konvex, Vorderrand kräftig gerandet, nicht konvex vorgezogen, wie der Seitenrand locker und kräftig behaart; Oberfläche dicht und fein punktiert, ohne mikroskopische Behaarung in den Punkten. Scutellum breit dreieckig, fein und mäßig dicht punktiert, längs der Mitte ohne glatte Linie.

Elytren im hinteren Drittel am breitesten, Außenwinkel an der Spitze leicht stumpf, Streifen mäßig, Nahtstreifen etwas kräftiger eingedrückt und fein, dicht punktiert, Intervalle eben, fein und dicht punktiert, äußere Intervalle und vereinzelt auch die mittleren mit wenigen einzeln stehenden, langen, abstehenden Haaren, an der Schulterbeule in Nähe der Epipleuralkante mit einem einzelnen, langen Haar, in der Punktur sonst nur miktoskopisch behaart; Spitzenrand häutig, mit schmalem Saum aus feinen Mikrohärchen; Epipleuralkante kräftig, endet an der äußeren Spitzenrundung der Elytren, Epipleuren bis zur äußeren Spitzenrundung kurz und mäßig dicht behaart.

Unterseite glänzend und kräftig, mäßig dicht punktiert, nur spärlich behaart, mit wenigen kräftigen Haaren auf Mesosternum und Metasternalplatte, Metacoxalplatten außen mit einigen kräftigen Haaren, Abdominalsternite mit je einer Querreihe haartragender Borstenpunkte zwischen der übrigen feinen, zerstreuten Punktur, vorletztes Sternit in der Mitte mit schmalem chitinösen Saum; Mesosternum zwischen den Mesocoxae etwas breiter als der Metafemur. Verhältnis: Metepisternen-/Metacoxalänge: 1/1,75, AuBenwinkel der Metacoxalplatten nicht abgerundet, Hinterrand gerade. Pygidium apikal bei beiden Geschlechtern schwach gewölbt, fein und mäßig dicht punktiert, am Apikalrand einzeln, lang behaart, sonst mikroskopisch behaart.

Mesofemur und Metafemur glänzend und mit zwei Längshaarpunktreihen, dazwischen nur sehr spärlich fein punktiert, Metafemur vorn scharf gekantet und breit, neben dem Vorderrand ohne krenulierte Linie, Punkte der vorderen Längspunktreihe einfach, deren Ränder nicht krenuliert, Punkte dex hinteren Längshaarpunktreihe dicht und kräftig, Hinterrand fast gerade, kahl, apikal weder ventral noch dorsal krenuliert, dorsoapikal zur Aufnahme der Tibia breit deckenartig produziert. Mesotibia an der 

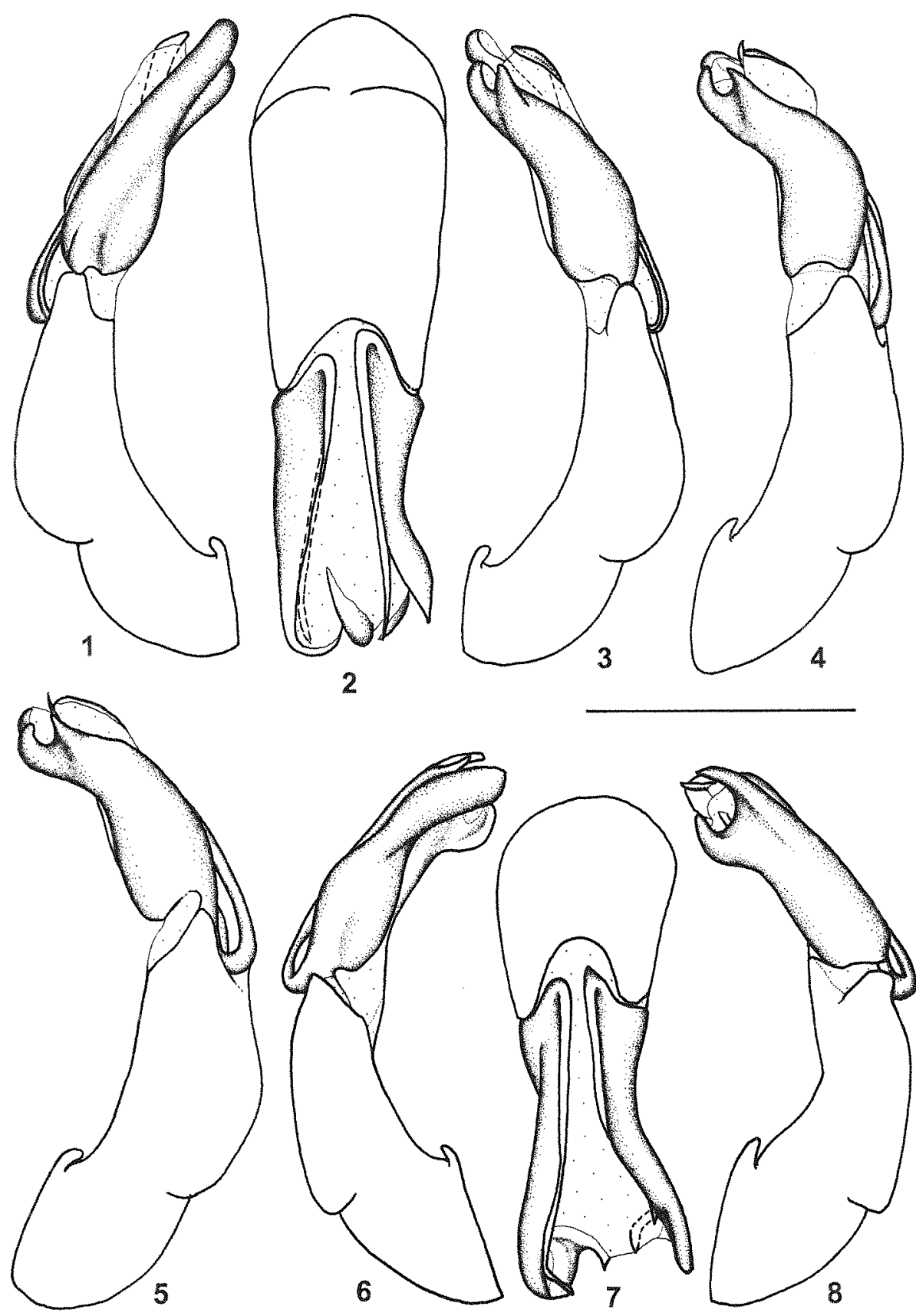

2

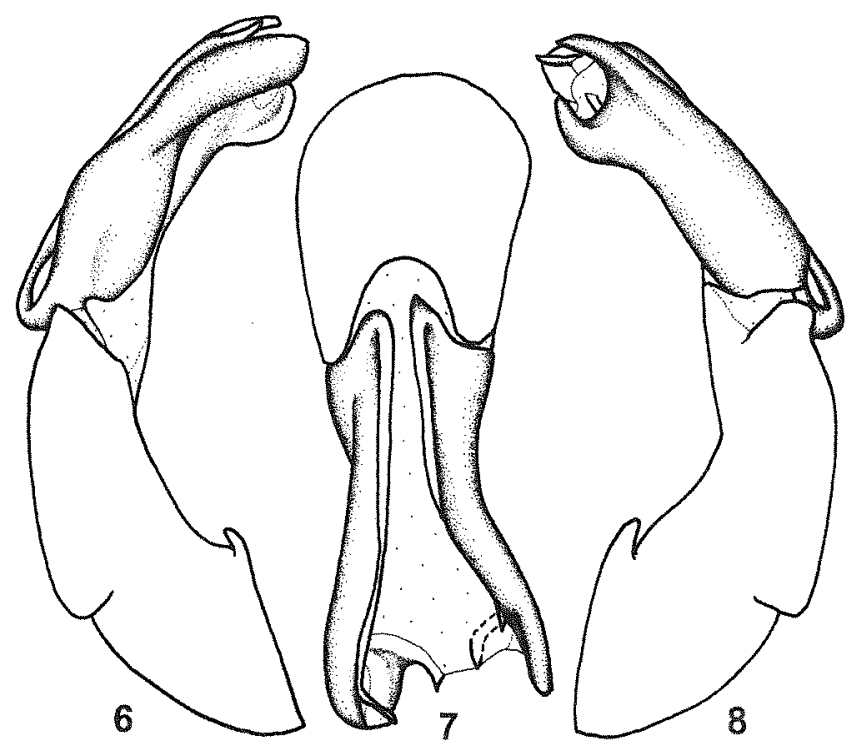

Fig. 1-8: 1-5: Maladera straba (BRENSKE), 1-3: Syntypus (A. apogonoides (BRENSKE)), Singapore/Collection Mniszeck" (MNHN), 4: Syntypus (M. straba (BRENSKE)), Ceylon, 5: Syntypus (A. stupida (BRENSKE)) Madagascar; 6-8: Maladera detersa (ERICHSON), Lectotypus „24901/detersa Er.* China Meyen." (ZMHB). Aedoeagus lateral $(1,3,4,5,6,8)$, Patameren dorsal $(2,7)$. Maßstab: $1 \mathrm{~mm}$. 
Ventralkante nur mit zwei feinen Borsten. Metatibien kurz und breit, in der Mitte am breitesten, basal verschmälert, Verhältnis: Breite/Länge: 1/2,4; dorsal scharf gekantet, mit zwei kräftigen Außendorngruppen, die basale kurz vor der Mitte, die apikale bei etwa 3/4 der Metatibienlänge, basal davor eine weitere kräftige Borste aber ohne krenulierte Leiste; außen schwach längsgewölbt, lateral sehr fein und spärlich punktiert und sonst kahl; Ventralkante mit fünf gehöckert stehenden, langen Borsten, die den gleichen Abstand zueinander haben; Innenseite völlig glatt und kahl, ventroapikad an der Tarsaleinlenkung seicht konkav ausgeschnitten. Tarsen dorsal unpunktiert, ohne Seitenleisten, Metatarsen ventral mit krenulierter Leiste und ventral kahl, unmittelbar daneben eine weitere kräftige Längskante, Metatarsomer 1 etwas kürzer als die zwei folgenden Segmente zusammen und nur wenig länger als der obere Enddorn der Metatibien; Protibia sehr kurz, 2-zähnig. Alle Klauen normal, symmetrisch.

Aedoeagus: Fig, 6-8.

Bemerkung: Maladera detersa unterscheidet sich von $M$. straba durch die weniger stark gebogene rechte Paramere und deren tiefere apikale kreisförmige Ausrandung, die stärker laterad verlagert und deutlich größer ist. Thr dorsaler Lobus ist deutlich länger als der ventrale. Im Gegensatz zu $M$. straba ist die linke Paramere leicht median gekrümmt.

Da die Originalbeschreibung keine Anzahl von vorliegenden Typenexemplaren liefert, wurde der einzige aufgefundene Syntypus als Lectotypus festgelegt.

\section{Literatur}

AHRENS, D. im Druck a: Monographie der Sericini des Himalaya (Coleoptera: Scarabaeidae). - Folia Heyrovskyana, Supplementum.

AHRENS, D. im Druck b: Maladera affinis (BLANCHARD, 1850) comb. n. (Coleoptera, Scarabaeoidea, Sericini), an oriental faunal element in the Malagasy region. - Deutsche Entomologische Zeitschrift.

Arrow, G. J. 1916: The Melolonthine Beetles of Ceylon. - Annals and Magazine of Natural History 8 (XVIII): $429-444$.

BRENSKE, E. 1898: Die Serica-Arten der Erde. - Berliner Entomologische Zeitschrift 43: 205-403.

BRENSKE, E. 1899: Sur quelques nouvelles especes de Melolonthides (gente Serica) de Cambodge et du

Siam appartenant au Museum de Paris. - Bulletin du Museum d'Histoire Naturelle, Paris 5: 414-416. BrensKe, E. 1900: Die Serica-Arten der Erde. IV. - Berliner Entomologische Zeitschrift 45: 39-96. BRENSKE, E. 1902: Die Serica-Arten der Erde. VII. - Berliner Entomologische Zeitschrift 47: 1-70. ERICHSON, W. 1834: Coleoptera. - In: MEYEN, F. J. F. : Beiträge zur Zoologie gesammelt auf einer Reise um die Erde. 6. Vol. 16 Supplement, Breslau, Bonn: 219-276.

Moser, J. 1915: Neue Serica-Arten. - Deutsche Entomologische Zeitschrift 1915: 337-393.

\section{Anschrift des Verfassers:}

\section{DIRK AHRENS}

Deutsches Entomologisches Institut

im Zentrum für Agrarlandschafts- und

Landnutzungsforschung (ZALF)

Schicklerstraße 5

D-16225 Eberswalde, Deutschland

e-mail: dahrens@zalf.de, ahrens.dirk_col@gmx.de 